\title{
C2 Segmental-Type Vertebral Artery Diagnosed Using Computed Tomographic Angiography
}

\author{
Myoung Soo Kim, M.D., Ph.D. \\ Department of Neurosurgery, National Medical Center, Seoul, Korea
}

Objective : Sometimes a vertebral artery (VA) enters the spinal canal via the C1-2 intervertebral space, a variation regarded as a $\mathrm{C} 2$ segmental-type VA. This paper describes the anatomy of the C2 segmental-type VA and reviews its clinical importance.

Methods : Between March 2014 and November 2015, 3386 patients underwent computed tomographic angiography. I identified C2 segmental-type VAs, associated vascular variation, the origin of ipsilateral posterior inferior cerebellar arteries (PICAs), and the clinical symptoms associated with C2 segmental-type VAs. The origin of an ipsilateral PICA is divided into 5 types. A type 1 PICA originates from ipsilateral VAs coursing suboccipitally (IVASO), a type 2 originates from ipsilateral proximal C2 segmental-type VAs, a type 3 originates from ipsilateral distal C2 segmental-type VAs. For type 4, the PICA does not originate from an ipsilateral VA. For type 5, the PICA is the terminal end of an ipsilateral C2 segmental-type VA.

Results : One hundred thirteen patients had $121 \mathrm{C} 2$ segmental-type VAs; 47 were associated with an IVASO, and 74 were not. Four type 1, 13 type 2, 60 type 3, 42 type 4, and two type 5 PICAs were identified. Only one patient showed symptoms associated with a C2 segmental-type VA, being a 71-year-old man presenting with a C2 segmental-type VA infarction.

Conclusion : For C2 segmental-type VAs, the ipsilateral IVASO and origin of the PICA are important for predicting the outcome of this type of VA infarction.

Key Words : Vertebral artery · Congenital abnormalities · Computed tomography angiography · Infarction.

\section{INTRODUCTION}

Normally, the V3 segment of the vertebral artery (VA) enters the subarachnoid space via the superior surface of the $\mathrm{Cl}$ posterior arch. Sometimes the VA enters the spinal canal via the C1-2 intervertebral space, a variation regarded as a C2 segmental-type $\mathrm{VA}^{4,6,8)}$. Extremely rarely, the VA enters the spinal canal via the intervertebral space of the $\mathrm{C} 2-3$ vertebrae, a variation regarded as $\mathrm{C} 3$ segmental-type $\mathrm{VA}^{18)}$.

In the present study, I reviewed C2 segmental-type VAs diagnosed using computed tomography (CT) angiography. I consider that a C2 or C3 segmental-type VA is the result of variations in the development of the posterior spinal artery and lateral spinal artery as proposed by Siclari et al. ${ }^{11)}$ and Lasjaunias et al. ${ }^{9)}$.

- Received : March 31, 2017 •Revised : May 13, 2017 •Accepted : June 9, 2017

- Address for reprints : Myoung Soo Kim, M.D., Ph.D.

Department of Neurosurgery, National Medical Center, 245 Eulji-ro, Jung-gu, Seoul 04564, Korea

Tel : +82-2-2260-7180, Fax : +82-2-2262-4876, E-mail : hanibalkms@hanmail.net

This is an Open Access article distributed under the terms of the Creative Commons Attribution Non-Commercial License (http://creativecommons.org/licenses/by-nc/4.0) which permits unrestricted non-commercial use, distribution, and reproduction in any medium, provided the original work is properly cited. 
Here, I report 121 C2 segmental-type VAs that were identified by CT angiography in 113 patients. This article describes the anatomy and embryological development of the $\mathrm{C} 2$ segmental-type VAs and reviews the clinical importance of this anomaly. I do not present anatomical points regarding $\mathrm{C} 2$ segmental-type VAs in occipitocervical fixation.

\section{MATERIALS AND METHODS}

Images obtained by $\mathrm{CT}$ angiography were analyzed for the presence of a C2 segmental-type VA. I excluded CT angiography performed on foreign patients or conducted outside our institution. The CT angiography was performed for a variety of reasons, including symptoms of cerebral ischemia, hemorrhagic contusion, intracerebral hemorrhage, headache, dizziness, sensory change, transient ischemic attack, and routine checkup. All images were evaluated by a single neurosurgeon.

CT angiography of the intracranial and extracranial vessels of 3386 patients (1880 females and 1506 males; age $58.73 \pm 15.58$ years; range, 11-94 years) was conducted between March 3, 2014 and November 12, 2015 and the results were analyzed. An Aquilion Prime 160-slice CT scanner (Toshiba Medical Systems, Tokyo, Japan) was used for 2454 patients, and an Aquilion CXL edition 128-slice CT scanner (Toshiba Medical Systems) was used for 932. After acquisition of nonenhanced CT data, contrast-enhanced CT angiography was performed. The parameters for the CT angiography acquisitions were as follows : $100 \mathrm{kVp}, 225 \mathrm{~mA}$, field-of-view $220 \mathrm{~mm}$, detector collimation $80 \mathrm{~mm} \times 0.5 \mathrm{~mm}$, table speed $25.5 \mathrm{~mm} /$ rotation, gantry rotation speed $0.75 \mathrm{~s} /$ rotation, reconstructed section thickness $0.5 \mathrm{~mm}$, and reconstruction increment $0.3 \mathrm{~mm}$ for the Aquilion Prime scanner; and $120 \mathrm{kVp}, 250 \mathrm{~mA}$, field-of-view 240 $\mathrm{mm}$, detector collimation $64 \mathrm{~mm} \times 0.5 \mathrm{~mm}$, speed $20.5 \mathrm{~mm} /$ rotation, gantry rotation speed $0.5 \mathrm{~s} /$ rotation, reconstructed section thickness $0.5 \mathrm{~mm}$, and reconstruction increment 0.5 $\mathrm{mm}$ for the Aquilion CXL scanner. The scan range extended from $2 \mathrm{~cm}$ below the aortic arch to a point $1 \mathrm{~cm}$ above the level of the lateral ventricles.

Using a power injector, we administered $100 \mathrm{~mL}$ of iopamidol (Pamiray 370, Dongkook Pharmaceuticals, Seoul, Korea) intravenously at $4.0 \mathrm{~mL} / \mathrm{s}$ via an 18-gauge catheter positioned in a peripheral vein, and the scan delay was individually adapted using a bolus-tracking technique. For the bolus tracking, first, a single nonenhanced low-dose scan of the level of the upper neck was made. When contrast material administration was commenced, repeated low-dose monitoring scans were made every second. When the contrast material was first seen in the common carotid artery, CT angiography was triggered automatically without any time delay. The data were transferred to a personal computer. Reconstructions of the images in 3D were obtained using commercially available software (Vitrea 2; Vital Images, Minnetonka, MN, USA). 3D CT angiographic images were reconstructed from the data using a volume-rendering technique. A series of 17 projection images at every $20^{\circ}$ around the cephalocaudal axis were generated and then transferred to a picture archiving and communication system.

I reviewed C2 segmental-type VAs, the origin of ipsilateral posterior inferior cerebellar arteries (PICAs), associated vascular anomalies, and the clinical symptoms associated with C2 segmental-type VAs. Ipsilateral PICAs originating from C2 segmental-type VAs between the C2 vertebral foramen and vertebrobasilar junction are divided into 5 types. A type 1 PICA originates from ipsilateral VAs coursing suboccipitally (IVA$\mathrm{SO}$ ), a type 2 originates from ipsilateral proximal C2 segmental-type VAs, and a type 3 originates from ipsilateral distal C2 segmental-type VAs. For type 4, the PICA does not originate from an ipsilateral VA, and a type 5 PICA is the terminal end of an ipsilateral C2 segmental-type VA (Fig. 1). I also reviewed bony abnormalities of the atlas and craniocervical junction in patients with a $\mathrm{C} 2$ segmental-type VA.

\section{RESULTS}

Of the 3386 patients who underwent CT angiography, 113 (68 females and 45 males; age $57.95 \pm 14.71$ years; range, $16-84$ years; $113 / 3386=3.3 \%$ ) had 121 C2 segmental-type VAs. I identified 39 right C2 segmental-type VAs, 66 left C2 segmentaltype VAs, and 8 bilateral C2 segmental-type VAs. Forty-seven C2 segmental-type VAs were associated with IVASO (Fig. 2) and 74 were not (Fig. 3).

Four PICAs originated from IVASOs (type 1), 13 PICAs originated from proximal C2 segmental-type VAs (type 2), 60 PICAs originated from distal C2 segmental-type VAs (type 3), 42 PICAs did not originate from an ipsilateral VA (type 4), and two PICAs were terminal ends of ipsilateral C2 segmentaltype VAs (type 5) (Fig. 4). In 42 type 4 cases, there was a contra- 


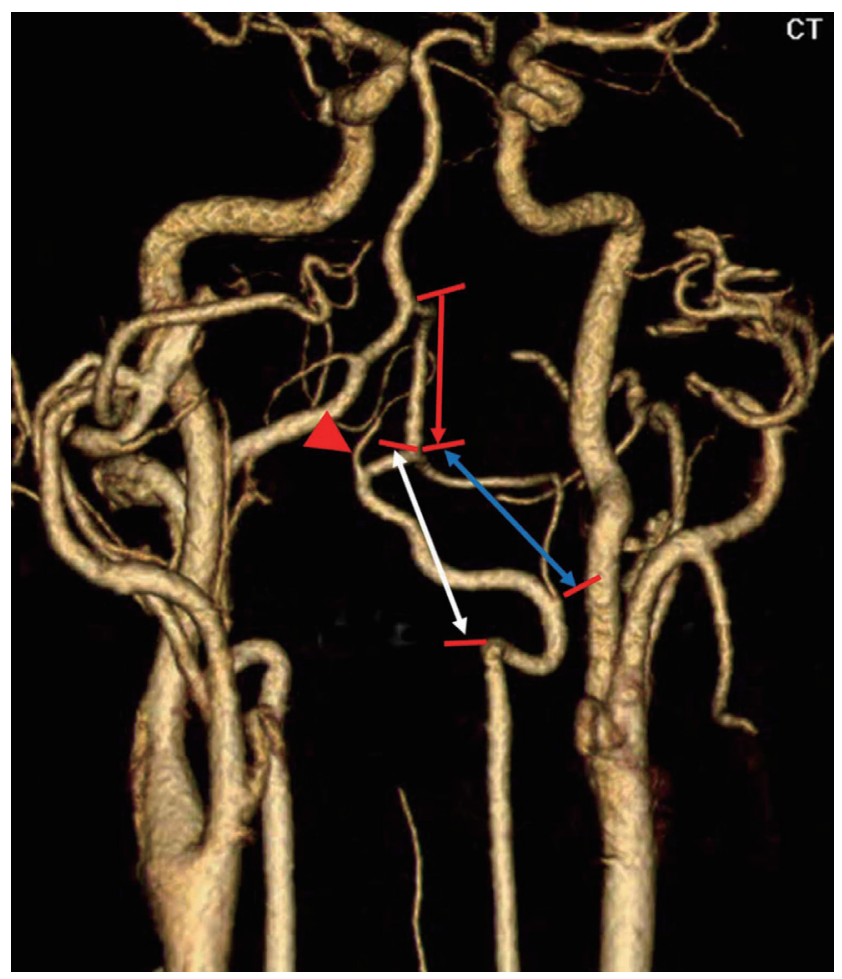

Fig. 1. Origins of the ipsilateral posterior inferior cerebellar artery (PICA) from the C2 segmental-type vertebral artery (VA). The type 1 PICA originates from the ipsilateral VA, coursing suboccipitally (ipsilateral VAs coursing suboccipitally, blue segment). The type 2 PICA originates from the ipsilateral proximal C2 segmental-type VA (white segment). The type 3 PICA originates from the ipsilateral distal VA (red segment). The type 4 PICA does not originate from the VA between the C2 vertebral foramen and the vertebrobasilar junction. In type 5, the PICA is the terminal end of the ipsilateral C2 segmental-type VA. The left PICA (red arrowhead) is type 2 .

lateral PICA in 18 cases, absent contralateral PICA and ipsilateral anterior inferior cerebellar artery (AICA) in 16 cases, absent contralateral PICA and contralateral AICA in four cases, and absent contralateral PICA and both AICA in four cases.

Among the 113 patients with C2 segmental-type VAs, I identified 26 vascular anomalies in 24 patients and four bony anomalies in four patients. Among these patients with $\mathrm{C} 2 \mathrm{seg}$ mental-type VAs, I found eight fenestrations (1 anterior communicating artery, one A2, three basilar arteries, three VAs), two azygos anterior cerebral arteries, one C3 segmental-type VA, eight left common carotid arteries originating from the right brachiocephalic trunk, six left VAs originating from the aortic arch, and one common trunk of the left subclavian and left common carotid artery. I found two bony defects of the posterior arch of the atlas, one occipitalization of the atlas, and one basilar impression.

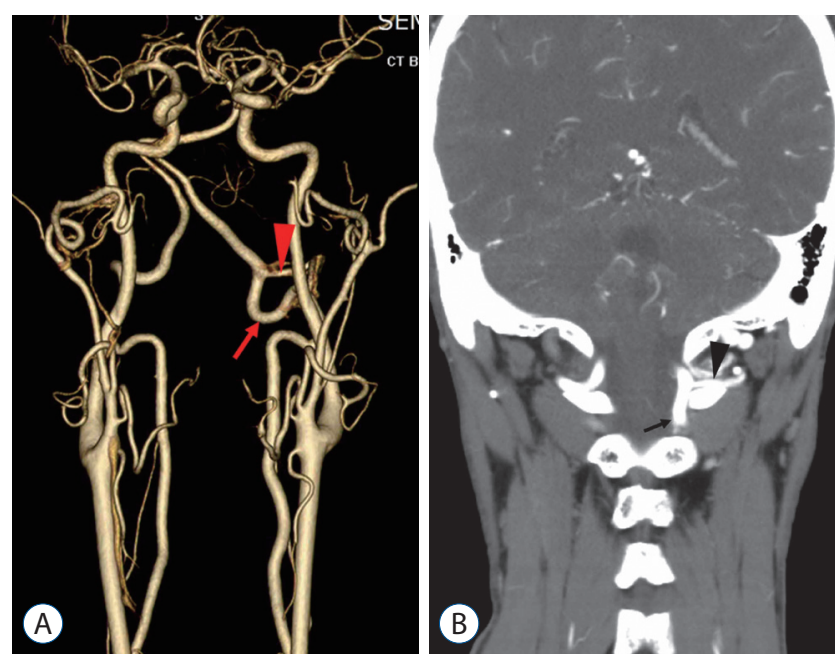

Fig. 2. Computed tomographic angiography of a 61-year-old man showing a left C2 segmental-type vertebral artery (VA, red arrow) with ipsilateral VA coursing suboccipitally (ipsilateral VAs coursing suboccipitally [IVASO], red arrowhead) (A). Coronal computed tomography showing the left C2 segmental-type VA (black arrow) entering the spinal canal between the $\mathrm{C} 1$ and $\mathrm{C} 2$ vertebrae and the IVASO (black arrowhead) entering the spinal canal between the atlas and occiput (B).
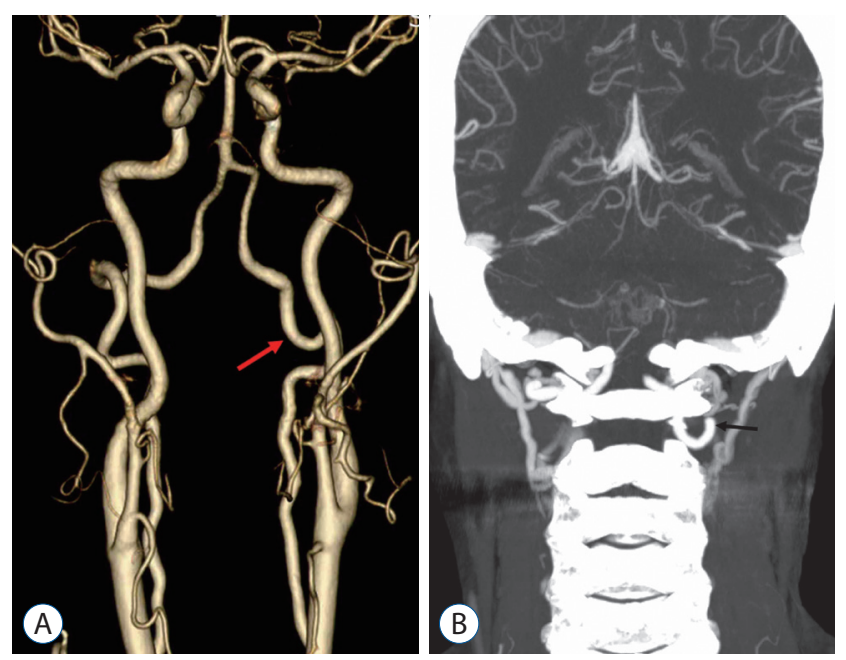

Fig. 3. Computed tomographic angiography of a 64-year-old man showing a left C2 segmental-type vertebral artery (red arrow, A). Coronal computed tomography showing the left vertebral artery entering the spinal canal between $\mathrm{C} 1$ and $\mathrm{C} 2$ (black arrow, B).

Only one patient showed symptoms associated with a C2 segmental-type VA, being a 71-year-old man presenting with a right $\mathrm{C} 2$ segmental-type VA infarction (Fig. 5). This patient presented with nausea, vomiting, and gait difficulty. Magnetic resonance angiography demonstrated no visible right VA except for a terminal segment. A diffusion-weighted image showed recent right lateral medullary and right cerebellar infarction be- 

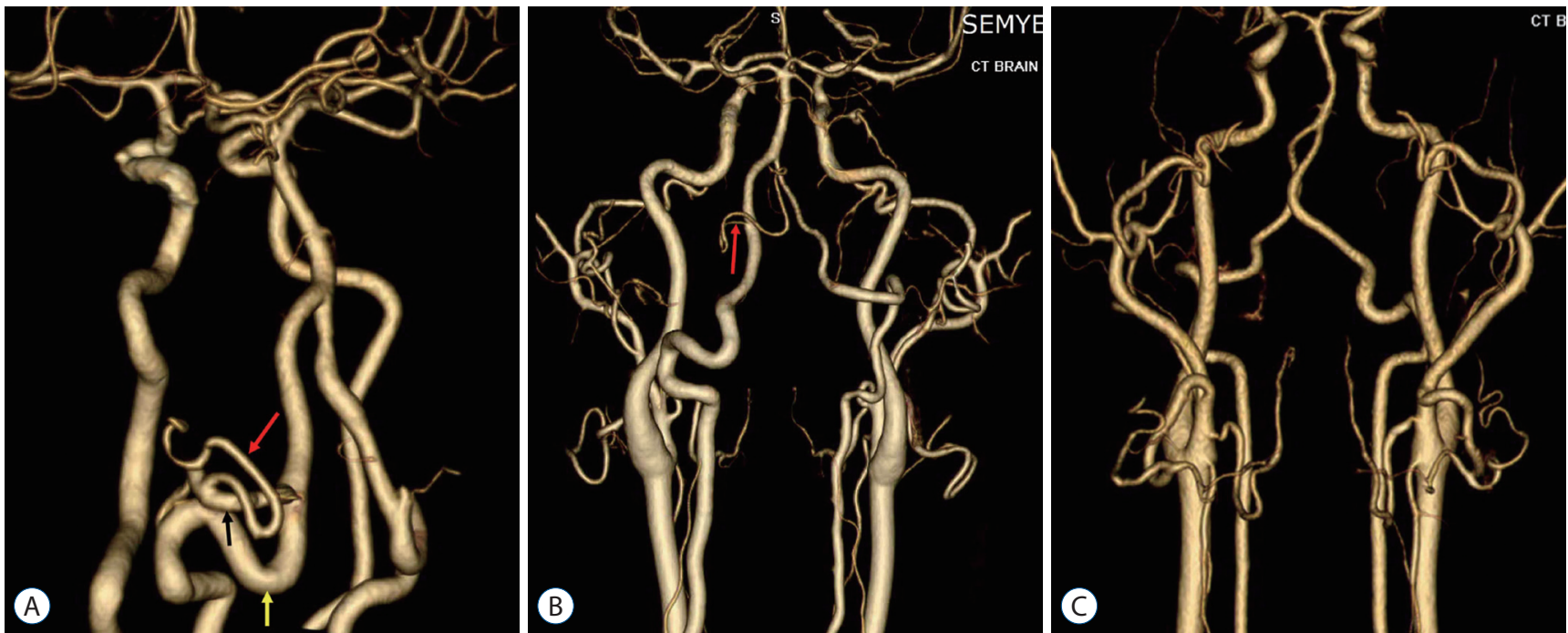

Fig. 4. Types of posterior inferior cerebellar arteries (PICAs) originating from a vertebral artery (VA). Type 1 (red arrow) originating from an ipsilateral VA coursing suboccipitally (black arrow; C2 segmental-type VA, yellow arrow) (A). Type 3 (red arrow) originating from a distal C2 segmental-type VA (B). C2 segmental-type vertebral artery with no PICA branch (type 4, C).
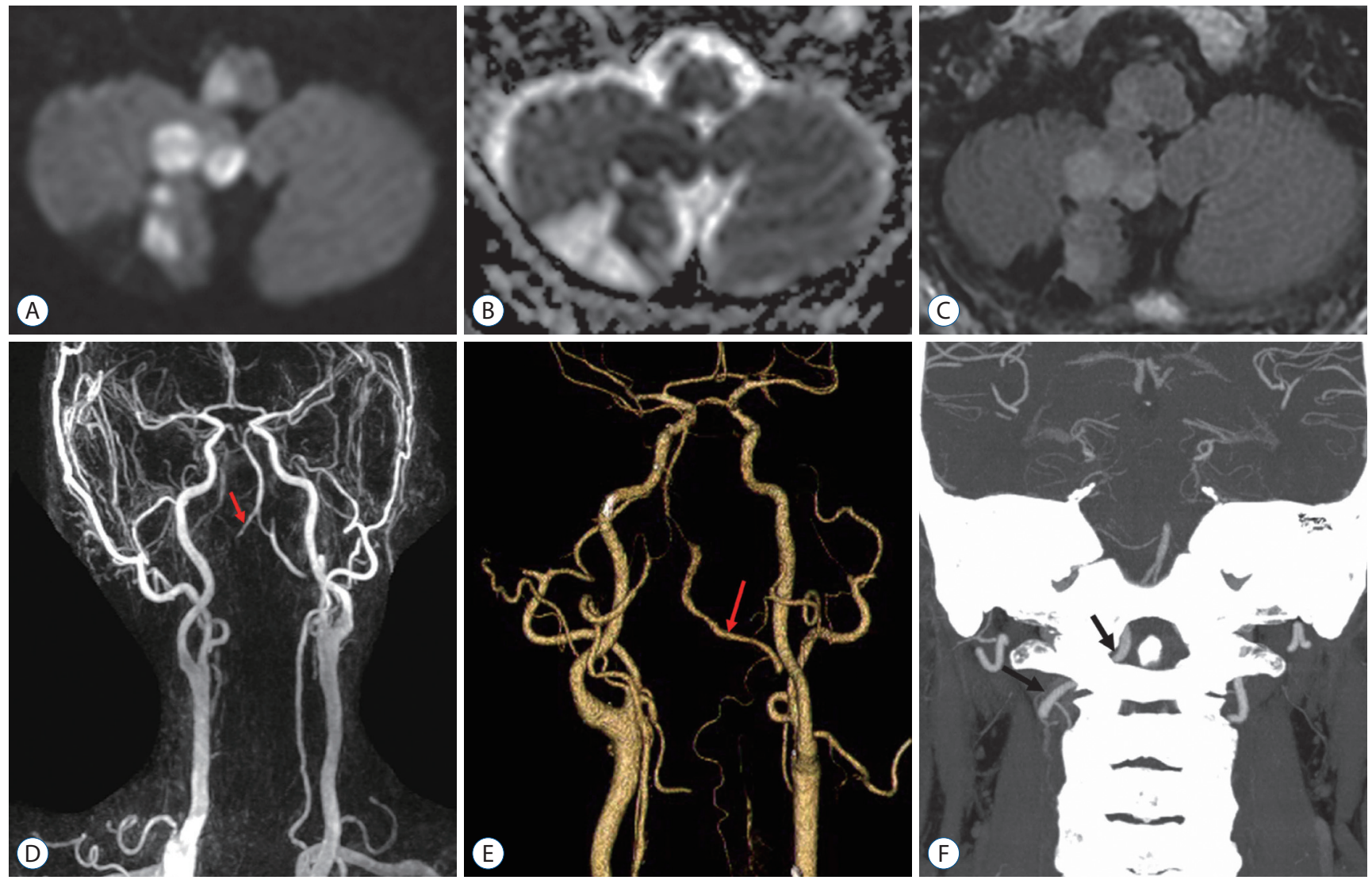

Fig. 5. Brain magnetic resonance images (A : diffusion-weighted image, B : apparent diffusion coefficient map, C : fluid-attenuated inversion-recovery image) showing an old right cerebellar infarction and a recent infarction involving the right cerebellum and right lateral medulla. Magnetic resonance angiography (D) demonstrating no sign of the right vertebral artery except for a terminal segment (red arrow). Computed tomographic angiography (E) performed 6 months later demonstrating a partially recanalized right C2 segmental-type vertebral artery (red arrow). Coronal computed tomography (F) showing a right $\mathrm{C} 2$ segmental-type vertebral artery (black arrow). 
cause of a right VA infarction and an old right cerebellar infarction. CT angiography and coronal CT performed 6 months later showed a partially recanalized right VA. The ipsilateral PICA was type 4. During 18 months of follow up, this patient showed no ischemic attack after maintaining Plavix (Clopidogrel Bisulfate, Handok, Seoul, Korea) 75 mg once per day and atorvastatin calcium (Lipitor, Pfizer Korea Corporate, Seoul, Korea) $10 \mathrm{mg}$ once per day. I did not find any symptom as a result of cervical cord or medullary compression because of a C2 segmental-type VA in any of the 113 patients with C2 segmental-type VAs.

\section{DISCUSSION}

\section{C2 segmental-type VA}

Researchers have suggested 2 possible developmental explanations for a C2 segmental-type VA. One is a persistent first intersegmental artery ${ }^{15,16)}$. If the first intersegmental artery persists without persistence of a normal VA branch, the VA takes an anomalous course and enters the spinal canal at the level between the $\mathrm{C} 1$ and $\mathrm{C} 2$ vertebral bodies ${ }^{15,16)}$. The second possible mechanism, proposed by Lasjaunias et al. ${ }^{9)}$, Siclari et al. ${ }^{11)}$, and $\mathrm{Kim}^{7}$, is that the intradural course of the distal VA at the C1-2 intervertebral space is related to variations in the size and connection of the lateral spinal artery or posterior spinal artery. I concur with this second mechanism.

I consider that persistent cervical intersegmental artery describing the C2 segmental-type VA is a misnomer. Persistent cervical intersegmental artery (CIA) was originally used to describe a persistent carotid connection. The segment of the VA proximal to a persistent CIA is generally absent ${ }^{7)}$. Indeed, hypoplasia of the lower vertebral segment of a VA is presumably the reason for the persistence of the embryonic anastomosis ${ }^{10)}$. In their textbook, Mayer and $\mathrm{Kier}^{10)}$ reported a persistent CIA originating from the common carotid artery (figures 24-30 of page 749 in Mayer and Kier $^{10)}$ ). In this case, a persistent CIA had no VA proximal segment. Failure of involution in one of the first 6 cervical intersegmental arteries causes a variety of abnormal origins of VAs. If a persistent CIA occurs in the upper (first to second) intersegmental arteries, the result is an abnormal origin of the VA from the internal or external carotid artery; if it occurs in the lower (third through sixth) cervical intersegmental arteries, the result is an abnormal origin of the
VA from the aortic arch or common carotid artery ${ }^{2}$.

In the present study, I found 121 cases of C2 segmental-type VAs and one case of a C3 segmental-type VA. These VAs enter the spinal canal via the C1-2 or C2-3 intervertebral space, respectively. A C3 segmental-type VA is extremely rare. Uchino et al. ${ }^{18)}$ reported two cases of C3 segmental-type VA, which were diagnosed by magnetic resonance angiography.

\section{Infarction or dissection of the C2 segmental-type VA}

A C2 segmental-type VA infarction or dissection is rare. I have experienced the single case described in the present article. To my knowledge, there are only three other cases reported in the literature ${ }^{1,5,19)}$. Bernard et al. ${ }^{1)}$ reported a case in a young man with a C2 segmental-type VA associated with an IVASO and type 3 PICA. Stroke resulted from C2 segmentaltype VA dissection in this patient who failed to respond to standard anticoagulation therapy (warfarin), but did well with angiographic coil obliteration of the C2 segmental-type VA. After coil occlusion of the VA, distal vertebral blood flow was maintained via an IVASO. In this case, the IVASO functioned as a collateral pathway after the occlusion.

Hayashi et al. ${ }^{5)}$ reported a case in a 69-year-old woman with a C2 segmental-type VA associated with an IVASO presenting with a subarachnoid hemorrhage because of a dissected IVASO. Vertebral angiograms on the 100th day after hemorrhage showed complete spontaneous occlusion of the dissected IVASO and collateral flow was maintained to the vertebrobasilar system via a C2 segmental-type VA with a type 2 PICA. In this case, the IVASO had no PICA branch.

Yoshida et al. ${ }^{19)}$ reported a case in a 52-year-old woman with a C2 segmental-type VA without IVASO presenting with recurrent embolic strokes. In this case, a left vertebral angiogram in the normal position showed a tortuous configuration of the C2 segmental-type VA without IVASO and a type 4 PICA. A left vertebral angiogram performed after rotating her neck rightward showed that the artery had slowly opacified and was severely stenosed with a pointed stump at the V3 portion. After applying a cervical collar to prevent neck rotation and prescription of an antiplatelet drug, she did not experience any attack.

From the review of these 3 cases and the case presented herein, I describe the outcome of treatment of C2 segmental-type VA infarction or dissection. In the case reported by Bernard et al. ${ }^{1)}$, the C2 segmental-type VA was associated with an IVASO 
and was the focus of the cerebral infarction. In this scenario, the first treatment option is antiplatelet or anticoagulation medication. The second treatment option is obliteration of the C2 segmental-type VA. However, obliteration of the C2 segmental-type VA may result in a brainstem perforator infarction. In the case reported by Hayashi et al. ${ }^{5}$, the patient had a type 2 PICA. IVASO was the focus of the subarachnoid hemorrhage. In this scenario, the first treatment option is obliteration of the IVASO. However, if the IVASO has a PICA branch, the occipital artery to the PICA anastomosis should be followed by IVASO obliteration. The PICA in the case reported by Yoshida et al. ${ }^{19)}$ was type 4 . In that scenario, a C2 segmental-type VA infarction may result in brainstem perforator sacrifice and distal embolic infarction. In C2 segmental-type VA infarction, knowledge of the anatomy of the ipsilateral IVASO and PICA is important for the treatment decision and to predict the outcome of infarction. In particular, a type 1 or type 2 PICA associated with a C2 segmental-type VA may result in a poor outcome after VA dissection.

\section{Cervical cord compression by a C2 segmental-type VA}

A C2 segmental-type VA in itself has no clinical importance. A C2 segmental-type VA is rarely symptomatic. Suboccipital neuralgia $^{17)}$, torticollis, cervical cord myelopathy ${ }^{3,4,12-14)}$, and arm pain and dysesthesia ${ }^{12)}$ have been reported in conjunction with this anomaly.

The VA at the C1 level usually runs forward, upward, and medially to pierce the dura as the VA enters the foramen magnum. However, VAs that penetrate the dura between the $\mathrm{Cl}$ and C2 levels pass dorsomedially and then run ventrally at the $\mathrm{C} 1$ level, resulting in compression of the spinal cord by vascular loops. Most cases associated with a C2 segmental-type VA do not present cervical cord compression because of a wide subarachnoid space at the C1-2 level. However, the arteriosclerotic dilatation and tortuosity of a C2 segmental-type VA might cause compression of the spinal cord. It is possible that arteriosclerotic changes and the hammering effect of the $\mathrm{C} 2$ segmental-type VA may explain the long history of progressive signs and symptoms ${ }^{4,13)}$. Kim et al. ${ }^{6}$ reported a case of occipital neuralgia caused by compression of the cord by a C2 segmental-type VA. The symptoms improved as the patient's hyperthyroidism was controlled, suggesting hemodynamic stress as a reason for the onset of the symptoms.

Takahashi et al. ${ }^{12)}$ reported that a patient with cervical my- elopathy because of compression of the cord by bilateral C2 segmental-type VAs had basilar impression, occipitalization of the atlas, and a defect in the posterior arch of atlas. Takahashi et al. ${ }^{12)}$ suggested that these findings indicated probable congenital bony abnormalities. Hasegawa et al. ${ }^{4)}$ reported that the patient with cervical myelopathy because of compression of the cord by a C2 segmental-type VA had mild basilar impression and a hypoplastic posterior arch of the atlas. Hasegawa et al. ${ }^{4}$ assumed that the associated hypoplasia of the atlas and mild basilar impression provoked actual stenosis of the spinal canal at the level of atlas.

Among the 121 vascular anomalies identified in the present study, I did not find any symptom because of compression of the spinal cord by a C2 segmental-type VA.

\section{CONCLUSION}

The intradural course of the distal VA at the C1-2 intervertebral space is related to variations in the size and connection of the lateral spinal artery or posterior spinal artery. In C2 segmental-type VA infarction, knowledge of the anatomy of the ipsilateral IVASO and PICA is important for treatment decisions and for predicting outcome.

\section{CONFLICTS OF INTEREST}

The authors have no financial conflicts of interest.

\section{INFORMED CONSENT}

This type of study does not require informed consent.

\section{- Acknowledgements}

This work was supported by research Grant from the National Medical Center.

\section{References}

1. Bernard TJ, Mull BR, Handler MH, Harned RK, Filley CM, Kumpe DA, et al. : An 18-year-old man with fenestrated vertebral arteries, recurrent 
stroke and successful angiographic coiling. J Neurol Sci 260 : 279-282, 2007

2. Chen CJ, Wang LJ, Wong YC : Abnormal origin of the vertebral artery from the common carotid artery. Am J Neuroradiol 19 : 1414-1416, 1998

3. Furumoto T, Nagase J, Takahashi K, Itabashi T, lai H, Ishige N : Cervical myelopathy caused by the anomalous vertebral artery. A case report. Spine (Phila Pa 1976) 21 : 2280-2283, 1996

4. Hasegawa T, Kubota T, Ito H, Yamamoto S : Symptomatic duplication of the vertebral artery. Surg Neurol $20: 244-248,1983$

5. Hayashi T, Hirose Y, Sagoh M, Murakami H : Spontaneous occlusion of ruptured vertebral artery dissection at the extradural fenestration associated with extradural origin of the posterior inferior cerebellar artery-case report. Neurol Med Chir (Tokyo) 40 : 164-168, 2000

6. Kim K, Mizunari T, Kobayashi S, Ishii S, Teramoto A : Occipital neuralgia caused by the compression of the fenestrated vertebral artery: a case report. No Shinkei Geka 27 : 645-650, 1999

7. Kim MS : Developmental anomalies of the distal vertebral artery and posterior inferior cerebellar artery: diagnosis by $\mathrm{CT}$ angiography and literature review. Surg Radiol Anat 38 : 997-1006, 2016

8. Lasjaunias $P$, Berenstein, $A$, ter Brugge, $K G$ : Arterial supply to the posterior fossa central nervous system in Lasjaunias P, Berenstein A, ter Brugge KG (eds) : Surgical Neuroangiography, Clinical vascular anatomy and variaions, ed 2. New York: Springer, Verlag Berlin Heidelberg, 2001, Vol 1, pp224-260

9. Lasjaunias P, Vallee B, Person H, Ter Brugge $K$, Chiu M : The lateral spinal artery of the upper cervical spinal cord. Anatomy, normal variations, and angiographic aspects. J Neurosurg 63 : 235-241, 1985

10. Mayer PL, Kier EL : The ontogeneti and phylogenetic basis of cerebrovascular anomalies and variants in Apuzzo MLJ (ed) : Brain surgery, complication avoidance and management. New York : Churchill Livingstone, 1993, Vol 1, pp709-760

11. Siclari F, Burger IM, Fasel JH, Gailloud P : Developmental anatomy of the distal vertebral artery in relationship to variants of the posterior and lateral spinal arterial systems. Am J Neuroradiol 28 : 1185-1190, 2007

12. Takahashi Y, Sugita S, Uchikado H, Miyagi T, Tokutomi T, Shigemori M : Cervical myelopathy due to compression by bilateral vertebral arteries-case report. Neurol Med Chir (Tokyo) 41 : 322-324, 2001

13. Takahashi T, Tominaga T, Hassan T, Yoshimoto T : Cervical cord compression with myelopathy caused by bilateral persistence of the first intersegmental arteries: case report. Neurosurgery $53:$ 234-237, 2003

14. Takei $H$, Sagae M, Chiba K, Ogino $T$ : The long-term follow-up of surgical treatment for cervical myelopathy with severe nape and upper arm pain caused by the anomalous vertebral artery: case report. Spine (Phila Pa 1976) 33 : E611-E613, 2008

15. Tokuda K, Miyasaka K, Abe H, Abe S, Takei H, Sugimoto S, et al. : Anomalous atlantoaxial portions of vertebral and posterior inferior cerebellar arteries. Neuroradiology 27 : 410-413, 1985

16. Tran-Dinh HD, Soo YS, Jayasinghe LS : Duplication of the vertebro-basilar system. Australas Radiol 35 : 220-224, 1991

17. Trimble C, Reeves A, Pare L, Tsai F : Vertebral artery anomaly causing C2 suboccipital neuralgia, relieved by neurovascular decompression. J Neuroimaging $23: 421-424,2013$

18. Uchino A, Saito N, Uemiya N, Sonada KI : Diagnosis of a C3 segmental type of vertebral artery by magnetic resonance angiography: report of two cases. Surg Radiol Anat 38 : 873-876, 2016

19. Yoshida T, Shiga K, Uraoka M, Yoshikawa K, Owada K, Nakagawa M : C2 segmental type of vertebral artery with recurrent embolic strokes. Cerebrovasc Dis 20 : 58-61, 2005 